EPJ Web of Conferences 38, 17003 (2012)

DOI: $10.1051 /$ epjconf/20123817003

(C) Owned by the authors, published by EDP Sciences, 2012

\title{
QGSM development for spallation reactions modeling
}

\author{
M.I. Baznat, ${ }^{\text {a }}$, S.E. Chigrinov ${ }^{\mathrm{b}}$, and K.K. Gudima ${ }^{\mathrm{c}}$ \\ Institute of Applied Physics of Academy of Sciences of Moldova, Kishinev, Moldova
}

\begin{abstract}
The growing interest in spallation neutron sources, accelerator-driven systems, $R \& D$ of rare isotope beams, and development of external beam radiation therapy necessitated the improvement of nuclear reaction models for both stand-alone codes for the analysis of nuclear reactions and event generators within the Monte Carlo transport systems for calculations of interactions of high-energy particles with matter in a wide range of energy and in arbitrary 3D geometry of multicomponent targets. The exclusive approach to the description of nuclear reactions is the most effective for detailed calculation of inelastic interactions with atomic nuclei. It provides the correct description of particle production, single- and double-differential spectra, recoil, and fission product yields. This approach has been realized in the Quark Gluon String Model (QGSM) for nuclear reactions induced by photons, hadrons, and high energy heavy ions. In this article, improved versions of the QGSM model and a corresponding code have been developed tested and bench marked against experimental data for neutron production in spallation reactions on thin and thick targets in the energy range from a few $\mathrm{MeV}$ to several $\mathrm{GeV} /$ nucleon.
\end{abstract}

\section{Introduction}

The QGSM [1-3] model describes properly the interactions of particles and ions with matter at energies far beyond the reactor diapason, from tens of $\mathrm{MeV}$ to several $\mathrm{GeV}$, where the experimental data are rather scarce so that the development of fast and reliable methods for simulation of transport of high-energy photons, pions neutrons, protons, and ions becomes the major challenge. Several Monte Carlo (MC) codes, like FLUKA, GEANT4, MARS15, MCNPX, PHITS, MRED, and SHIELD have been developed for simulation of transport of high-energy hadrons and ions in numerous applications of high energy physics, accelerator science, external beam radiation therapy, space exploration program, etc. In calculations of interactions of high-energy particles with matter the central factor is the accurate description of photon-, hadron and nucleus-nucleus inelastic interactions in a wide range of energy and atomic mass. The exclusive approach to the description of nuclear reactions is the most effective for detailed calculation of inelastic interactions. It provides the correct description of particle production, single- and double-differential spectra, recoil, and fission product yields while fulfilling the momentum-energy conservation for each simulated event. In present the LAQGSM03.03 version [3] of QGSM is used for the analysis of nuclear reactions and as event generators within the different Monte Carlo transport systems. The development of radiation therapy and the growing interest in accelerator-driven systems and spallation neutron sources necessitates the improvement of nuclear models serving as event generators in radiation transport codes. The main objects for present improvements in the new version of QGSM code were:

\footnotetext{
a e-mail: baznat@theor.jinr.ru

b e-mail: serghei.chigrinov@phys.asm.md

c e-mail: gudima@theor.jinr.ru
}

- new compilations and parametrization of elastic, inelastic, and total $\mathrm{hN}$ and $\pi \mathrm{N}$ cross sections in the above energy range;

-more precise and effective model for calculation of inelastic NN, $\pi \mathrm{N}$ and $\gamma \mathrm{N}$ interactions with formation of a shortlived $\Delta$ and $\mathrm{N}^{*}$-resonances followed by its decay into a pion and a nucleon;

- fast MC algorithms for calculation of these interactions;

- formation of light nuclear fragments in the coalescence model, emission of photons on the de-excitation stage of reaction, and a more accurate description of the competition between the processes of particle evaporation, light nucleus evaporation and fission;

- more precise approach to modeling high-energy hadronhadron interactions, $\gamma \mathrm{N}$ and $\gamma \mathrm{d}$ interactions;

- implementation of new code as event generator into transport code SHIELD [4] for calculation of interactions of hadron and heavy ion beams with thick targets. This allows us to perform the analysis of the most important aspect of spallation reaction - neutron production from interactions of proton, pion, antiproton, $\mathrm{H}^{2}$ and, $\mathrm{He}^{3}$ with thin and thick $\mathrm{Ag}, \mathrm{Au}, \mathrm{Ta}, \mathrm{Pb}, \mathrm{Bi}, \mathrm{U}$ targets in the energy range from $\sim 0.5$ $\mathrm{GeV}$ up to $5.0 \mathrm{GeV}$.

\section{Modification of evaporation and fission in QGSM code}

In the modified version of QGSM code the decay probability for the emission of a particle $j$ from a parent compound nucleus $i$ is calculated similarly GEM [5] and for total decay width is given by

$$
\Gamma_{j}=\frac{\left(2 s_{j}+1\right) \mu_{j}}{(\pi \hbar)^{2} \rho_{i}(E)} \int_{V}^{E-Q_{j}} \sigma_{i n v} \epsilon \rho_{d}\left(E-Q_{j}-\epsilon\right) d \epsilon .
$$


The total radiation probability is

$$
\Gamma_{\gamma}\left(E^{*}\right)=\frac{3}{(\pi \hbar c)^{2}} \times \int_{0}^{E^{*}} \sigma_{\gamma}\left(\epsilon_{\gamma}\right) \frac{\rho\left(E^{*}-\epsilon_{\gamma}\right)}{\rho\left(E^{*}\right)} \epsilon_{\gamma}^{2} d \epsilon_{\gamma}
$$

The analysis shows that the increase in the emission of $\gamma$-quanta occurs near to binding energy of neutrons. This effect manifests itself stronger with growth of the parameter of level density. For high excitation energy the $\gamma$-emission is negligible in comparison with the particle emission, and it becomes important only at the energies around and below the particle separations energies. The evaporation process competes with another equilibrium process, i.e. with a fission. The fission probability, obtained from the Bohr and Wheeler theory is proportional to the level density $\rho_{f i s}(\epsilon)$ at the saddle point

$$
\Gamma_{F}=\frac{1}{2 \pi} \int_{0}^{E^{*}-B_{f i s}} \frac{\rho_{f i s}\left(E^{*}-B_{f i s}-\epsilon\right)}{\rho\left(E^{*}\right)} d \epsilon
$$

where $B_{f i s}$ is the fission barrier height. The level density $\rho(E)$ is calculated according to the Fermi-gas model using formula [6]

$$
\rho(E)=\frac{\pi}{12} \frac{\exp (2 \sqrt{a(E-\delta)})}{a^{1 / 4}(E-\delta)^{5 / 4}} \text { for } E>E_{x},
$$

where $a$ is the level-density parameter and $\delta$ is the pairing energy in MeV. The level density is calculated using Eq. (4) only for high excitation energies, $E \geq E_{x}$, where $E_{x}=$ $U_{x}+\delta$ and $U_{x}=2.5+150 / A_{d}$ (all energies are in $\mathrm{MeV}$ ). At lower excitation energies

$$
\rho(E)=\frac{\pi}{12} \frac{1}{T} \exp \left(\left(E-E_{0}\right) / T\right),
$$

where $T$ in the nuclear temperature defined as $1 / T=$ $\sqrt{a / U_{x}}-1.5 / U_{x}$. To provide a smooth connection of Eqs. (4) and (5) at $E=E_{x}, E_{0}$ is defined as $E_{0}=E_{x}-T(\log T-$ $\left.0.25 \log a-1.25 \log U_{x}+2 \sqrt{a U_{x}}\right)$. For the level density parameter $a$, we consider different sets of parameters of the form $a_{j}\left(Z, N, E^{*}\right)$, each with three empirically determined parameters,

$$
a\left(Z, N, E^{*}\right)=\tilde{a}(A)\left\{1+\delta W_{g s}(Z, N) \frac{f\left(E^{*}-\Delta\right)}{E^{*}-\Delta}\right\},
$$

where

$$
\tilde{a}(A)=\alpha A+\beta A^{2 / 3} B_{s}
$$

is the asymptotic Fermi-gas value of the level density parameter at high excitation energies. The function $f(E)$ is the following:

$$
f(E)=1-\exp (-\gamma E) .
$$

if $E^{*} \leq \Delta$, the limiting value of $a\left(E^{*}\right)$ is given by

$$
\lim _{E^{*}-\Delta \rightarrow 0} a\left(E^{*}\right)=\tilde{a}[1+\gamma \delta W] .
$$

In the previous LAQGSM03.03 version for emission of particles and process of fission the Generalized Evaporation Model (GEM) of Furihata [5] was used, in which probabilities of emission and fission are calculated in approach where the level density has a simple dependence
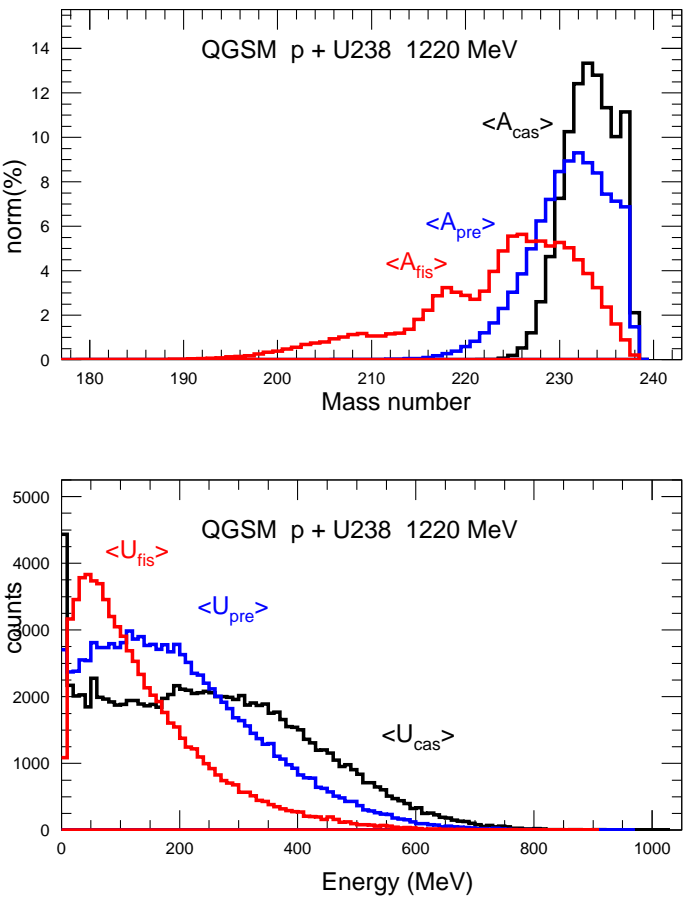

Fig. 1. The mass number and excitation energy distributions for different stages of reactions $\mathrm{p}+{ }^{238} \mathrm{U}$.

on excitation energy of a nucleus and the parameters are independent on the energy. This approach allows to obtain the analytical expressions for the widths of emission and fission, but it is justified at low excitation energy only. In the present version we use for level density the expression from [6] with parameters by Ignatyuk et al. [7], therefore all widths are calculated numerically. In addition, we refuse to relate the fission probability to neutron emission using the Atchison parametrization [8] for $\Gamma_{f} / \Gamma_{n}$. Fission is considered as one of channels in competition with emission of particles and light fragments. Our analysis shows that at small excitation energies, near neutron separation energy, the emission of $\gamma \mathrm{s}$ is important, therefore this channel was included with the expression for the emission width from [9]. As an example of the model application, we present the mass number and excitation energy distributions for different stages of reaction $\mathrm{p}+{ }^{238} \mathrm{U}$ at $E_{p}=1220 \mathrm{MeV}$ (figure 1). Figure 2 shows the experimental [10] and calculated relative mass yields $\mathrm{Y}(\%)$ in the reaction $\mathrm{n}+{ }^{238} \mathrm{U}$ at neutron energies $E_{n}=13,28,50,100,160 \mathrm{Mev}$. Contributions from a symmetric(green), antisymmetric(blue) modes are specified. The agreement between experimental data and calculated results is rather good. In this figure the mass distribution of final fission fragments after post-fission neutron emission is shown as well.

\section{Nuclear reactions on thin and thick targets}

In this section, a comparison of calculated neutron yields and neutron multiplicity distributions with an experimental data are presented. In case of thick targets, for more accu- 


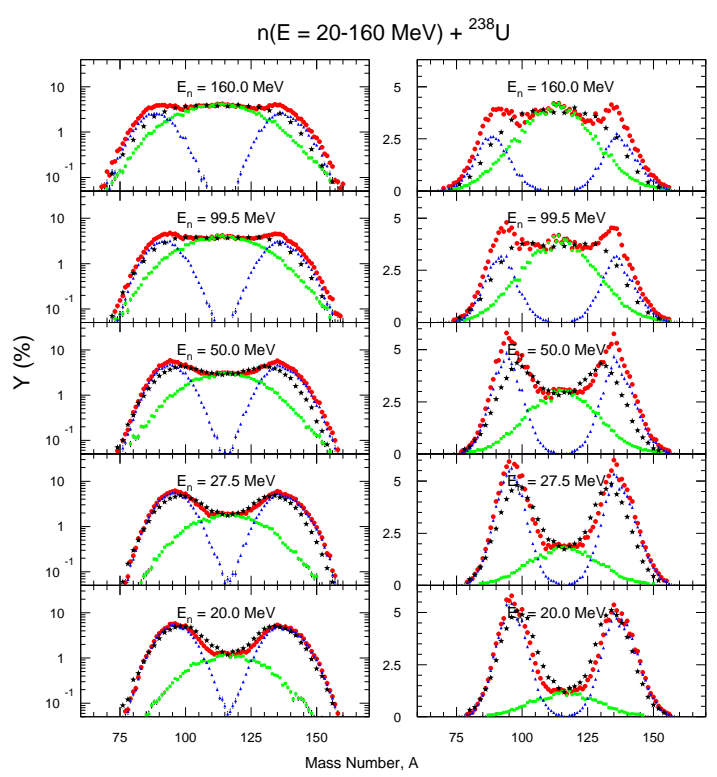

Fig. 2. Experimental relative mass yields of fragments(black circles) from neutron-induced fission of ${ }^{238} \mathrm{U}[10]$ and the results of calculations in logarithmic scale (left) and linear scale (right). Contributions from a symmetric (green) and antisymmetric (blue) modes are specified.

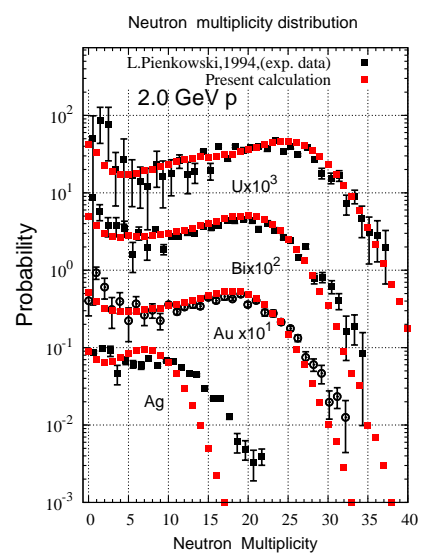

Fig. 3. Neutron multiplicity distributions for $2.0 \mathrm{GeV}$ protoninduced reactions on thin targets of $\mathrm{Ag}, \mathrm{Au}, \mathrm{Bi}$, and $\mathrm{U}$. The measurements [11-13] are shown by the black square, and the red ones are the results of our calculations not corrected for the detector efficiency.

rate simulation of nuclear reactions the new code was implemented as event generator into the well known Monte Carlo Transport code SHIELD to allow for secondary reactions, production and transport of secondary particles (n, $\mathrm{p}, \pi, \mathrm{K}, \mathrm{D}, \mathrm{H}^{3}, \mathrm{He}$ ). Calculation of neutron production and neutron multiplicity distributions have been performed for proton, pion $\left(\pi^{+}, \pi^{-}\right)$, antiproton $\left(\mathrm{p}^{-}\right), \mathrm{H}^{2}, \mathrm{He}^{3}$ - induced spallation/fission reaction in the energy range from $\sim 0.5$ $\mathrm{GeV}$ up to $5.0 \mathrm{GeV}$ on $\mathrm{Ag}$, $\mathrm{Au}, \mathrm{Ta}, \mathrm{Pb}, \mathrm{Bi}, \mathrm{U}$ targets of various geometries. The most detailed measurements of neutron multiplicity distributions were carried out in [11-13] with a $4 \pi$ neutron detector with the mean efficiency $\epsilon$ about $70-80 \%$ [13].

Figures 3 and 4 show the results of simulations for neutron multiplicity distributions performed with from 2.0

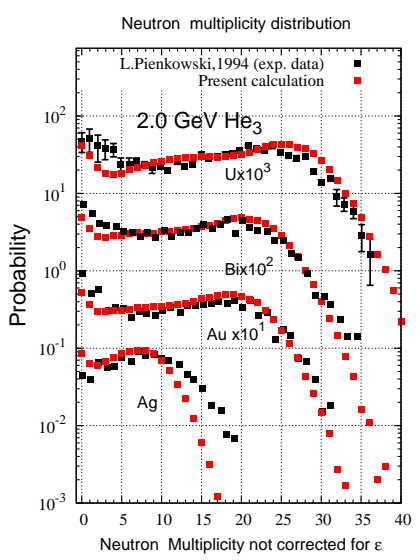

Fig. 4. Same as in figure 3 but for $\mathrm{He}^{3}$ with the energy $2.0 \mathrm{GeV}$.

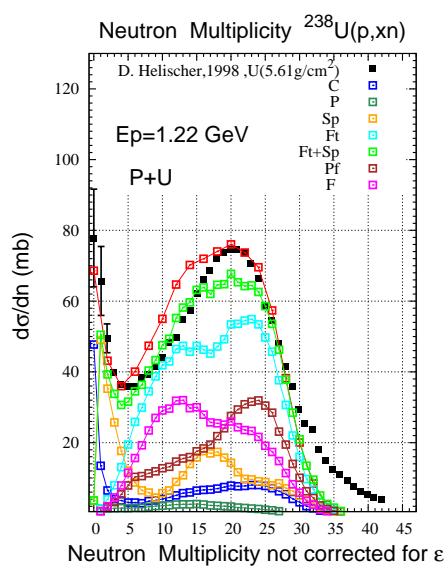

Fig. 5. Contributions of cascade (C), preequilibrium (P), evaporation without fission $(\mathrm{Sp})$ and fission neutrons (pre-fission $(\mathrm{Pf})$, post-fission $(\mathrm{F})$, and their sum $(\mathrm{Ft})$ ) to the total neutron multiplicity distribution (red symbols) for $1.22 \mathrm{GeV}$ proton- induced reaction on ${ }^{238} \mathrm{U}$ thin target.

$\mathrm{GeV}$ proton- and $2.0 \mathrm{GeV} \mathrm{He}^{3}$ - induced reactions on $\mathrm{Ag}$, $\mathrm{Au}, \mathrm{Bi}, \mathrm{U}$ thin targets.

Figure 5 displays the contributions of cascade, preequilibrium, and evaporation neutrons to the total neutron multiplicity distribution for $1.22 \mathrm{GeV}$ proton- induced reaction on the ${ }^{238} \mathrm{U}$ thin target. It can be seen that for thin targets the calculated neutron multiplicity distributions agree rather well with experimental ones. It is of significant interest for further development of QGSM to carry out comparison of neutron multiplicity distributions in reactions on thick targets, induced by high energy pions and antiprotons, with experimental data [11].

The results of our calculations of total neutron yield $(\mathrm{n} / \mathrm{p})$ from $\mathrm{Be}, \mathrm{Sn}$, and $\mathrm{Pb}$ thick targets irradiated with proton beam are compared with various measured data in the energy range from 0.3 to $5 \mathrm{GeV}$ (see figure 6). One can see a reasonably good agreement of the QGSM + SHIELD results with experimental data [14-17].

Figure 7 illustrates a comparison between experimental and calculated mean neutron multiplicities for a uranium target (the cylinder of $8-\mathrm{cm}$ diameter and $40-\mathrm{cm}$ long) and a lead target $(15-\mathrm{cm}$ diameter and $35-\mathrm{cm}$ long) with incident $p, \pi^{+}, \pi^{-}, K^{+}, D$ and $p^{-}$, in the energy range from 1.0 $\mathrm{GeV}$ to $6 \mathrm{GeV}$. It is seen that a combination QGSM, as event generator, and transport code SHIELD allows to get 


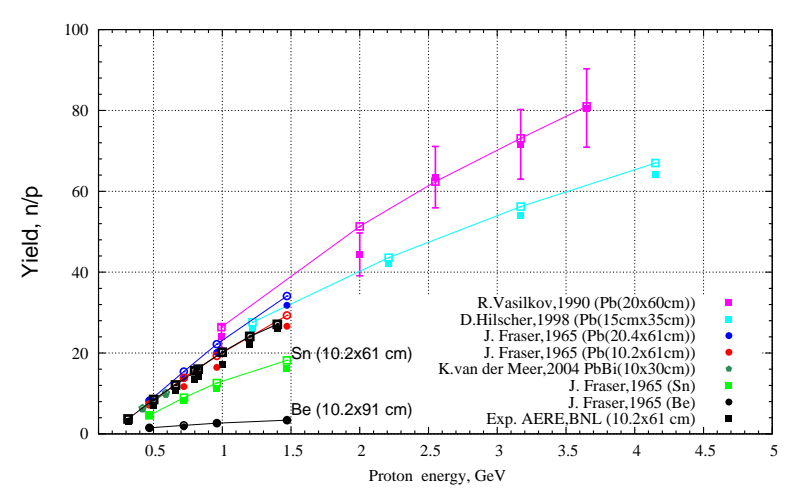

Fig. 6. Calculated and measured [14-17] total neutron yields (n/p) for $\mathrm{p}+\mathrm{Pb}, \mathrm{Sn}$, and Be thick targets at energy $E_{p}$. The curves connect the data points calculated with the QGSM + SHIELD transport code (open symbols). The filled symbols are the experimental data.

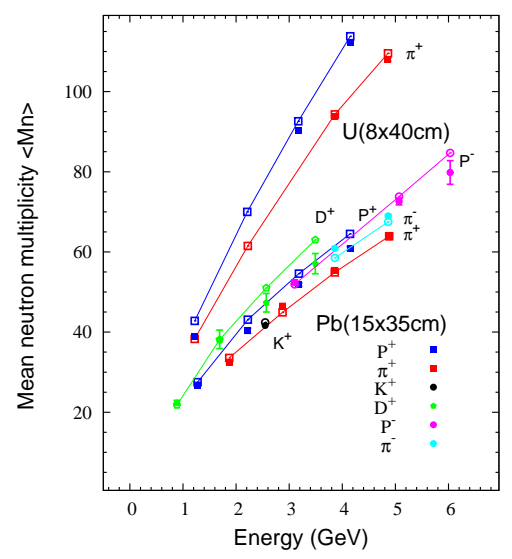

Fig. 7. Calculated and measured $[11,12]$ mean neutron multiplicities $<M_{n}>$ for incident $\mathrm{p}, \mathrm{p}^{-}, \pi^{+}, \pi^{-}, K^{+}, D^{+}$vs. incident kinetic energy on $\mathrm{U}$ target $(8 \mathrm{~cm}$ diameter and $40 \mathrm{~cm}$ long) and $\mathrm{Pb}$ target $(15 \mathrm{~cm}$ diameter and $35-\mathrm{cm}$ long). The curves connect the data points calculated with the QGSM + SHIELD transport code (open symbols). The filled symbols represent experimental data.

accurate description of neutron yields and neutron multiplicity distributions for both the thick targets.

\section{Conclusions}

Improved versions of the QGSM model and a corresponding code were developed for simulation of photon, hadron and nucleus-nucleus reactions in the energy range from the threshold to $5000 \mathrm{MeV} /$ nucleon. All modifications were implemented into new QGSM code which has been tested and bench marked against available experimental data. We successfully attempted to implement our new code into SHIELD transport code and analyzed the experimental data for neutron multiplicity distributions for interactions of proton, antiproton, $\pi^{+}, \pi^{-}, K^{+}$, and $D$ beams with thick targets of uranium and lead. The results of calculations are in good agreement with available experimental data in a large energy range of main interest for nuclear applications dealing with development of ADS systems.
The new code will be available for incorporation as an event generator into the GEANT4, MARS15, MCNPX, MRED, and SHIELD transport codes, employed in medical physics and radiation therapy at proton and ion accelerators, to study, among other things, nuclear reactions responsible for Single-Event Effects (SEE) in microelectronics. The code will be used in the E\&T RAW (Energy and Transmutation of Radioactive Wastes) project at Joint Institute for Nuclear Research (Dubna, Russia) for accurate simulation of spallation and fission reactions induced by hadrons, heavy ions.

The code allows to calculate the yields, energy spectra, and angular distributions of protons, neutrons, pions $\left(\pi^{-}, \pi^{0}, \pi^{+}\right), D,{ }^{3} \mathrm{H},{ }^{3} \mathrm{He},{ }^{4} \mathrm{He}$, fragments, and residual nuclei, radiation damage cross-sections, displacement atom cross-sections which are needed for production of radioisotopes for nuclear medicine, and in other domains of the applied nuclear physics and nuclear engineering.

This work was carried out under support of the Grant STCU (grant No.5238).

\section{References}

1. N.S. Amelin, K.K. Gudima, and V.D. Toneev, Sov. J. Nucl. Phys. 51, 327 (1990); 52, 172 (1990)

2. S.G. Mashnik, A.J. Sierk, K.K. Gudima, and M.I. Baznat, J. Phys.: Conf. Series 41, 340 (2006)

3. S.G. Mashnik, K.K. Gudima, N.V. Mokhov, and R.E. Prael, arXiv:0709.1736 [nucl-phys].

4. A.V. Dementyev and N.M. Sobolevsky, Rad. Meas. 30, 553 (1999)

5. S. Furihata, Nucl. Instr. Meth. B 171, 252 (2000)

6. A. Gilbert and A.G.W. Cameron, Can. J. Phys. 43, 1446 (1965)

7. A.V. Ignatyuk, G.N. Smirenkin, and A.S. Tishin, Sov. J. Nucl. Phys. 21, 255 (1975)

8. F. Atchison, Nucl. Instr. Meth. B 259, 100 (1998)

9. A.S. Iljinov, et al., Nucl. Phys. A 543, 517 (1992)

10. C.M. Zoller, Ph. D. Thesis, TU Darmstadt, Germany, (1997)

11. D. Hilscher, et al., Nucl. Instr. Meth. A 414, 100 (1998)

12. L. Pienkowski et al., Phys. Rev. C 56, 1909 (1997); Phys. Lett. B 336, 147 (1994)

13. J. Galin and U. Jahnke, J. Phys. G: Nucl. Part. Phys. 20, 1105 (1994)

14. R.G. Vassilkov and V.I. Yurevich, Proc. ICANSXI, Tsukuba, 340 (1990)

15. J.S. Fraser et al., Phys. Can. 21, 21 (1966); T.W. Armstrong et al., Nucl. Instr. Meth. 22, 540 (1984)

16. K. van der Meer et al., Nucl. Instr. Meth. B 217, 202 (2004)

17. M. Zucker, et al., Nucl. Sci. Eng. 129, 180 (1998) 